J. Austral. Math. Soc. 19 (Series B), (1975), 1-29.

\title{
SOME HIGH-ORDER ZERO-FINDING METHODS USING ALMOST ORTHOGONAL POLYNOMIALS
}

\author{
RICHARD P. BRENT
}

(Received 11 October 1974)

(Revised 19 February 1975)

\begin{abstract}
Some multipoint iterative methods without memory, for approximating simple zeros of functions of one variable, are described. For $m>0, n \geqq 0$, and $k$ satisfying $m+1 \geqq k>0$, there exist methods which, for each iteration, use one evaluation of $f, f^{\prime}, \cdots, f^{(m)}$, followed by $n$ evaluations of $f^{(k)}$, and have order of convergence $m+2 n+1$. In particular, there are methods of order $2(n+1)$ which use one function evaluation and $n+1$ derivative evaluations per iteration. These methods naturally generalize the known cases $n=0$ (Newton's method) and $n=1$ (Jarratt's fourth-order method), and are useful if derivative evaluations are less expensive than function evaluations. To establish the order of convergence of the methods we prove some results, which may be of independent interest, on orthogonal and "almost orthogonal" polynomials. Explicit, nonlinear, Runge-Kutta methods for the solution of a special class of ordinary differential equations may be derived from the methods for finding zeros of functions. The theoretical results are illustrated by several numerical examples.
\end{abstract}

\section{Introduction}

Traub [32] and Jarratt [13] have considered multipoint iterative methods for approximating a simple zero of a function $f$ which is more difficult to evaluate than its derivative $f^{\prime}$. (Examples of such functions are given in Sections 8 and 9.) Jarratt improved Traub's results by giving a fourth-order method which, for each iteration, uses one evaluation of $f$ and two of $f^{\prime}$, and is "without memory" in the sense of Traub [32]. This is rather surprising, for the obvious method [evaluating $f\left(x_{0}\right)$ and $f^{\prime}\left(x_{0}\right)$, computing the Newton-Raphson approximation $\tilde{x}_{0}=x_{0}-f\left(x_{0}\right) / f^{\prime}\left(x_{0}\right)$, evaluating $f^{\prime}\left(\tilde{x}_{0}\right)$, and taking $x_{1}$ as the appropriate zero of the quadratic $Q(x)$ which satisfies $Q\left(x_{0}\right)=f\left(x_{0}\right), Q^{\prime}\left(x_{0}\right)=$ $f^{\prime}\left(x_{0}\right)$, and $\left.Q^{\prime}\left(\tilde{x}_{0}\right)=f^{\prime}\left(\tilde{x}_{0}\right)\right]$ has order three rather than four. Jarratt showed that order four is attainable if we evaluate $f^{\prime}\left(\left(x_{0}+2 \bar{x}_{0}\right) / 3\right)$ instead of $f^{\prime}\left(\tilde{x}_{0}\right)$. For methods with two function evaluations and one derivative evaluation per iteration the results are less surprising: Ostrowski [29] showed that order four is attainable by evaluating $f\left(x_{0}\right), f^{\prime}\left(x_{0}\right)$, and $f\left(\tilde{x}_{0}\right)$.

(C) Copyright Australian Mathematical Society 1975

Copyright. Apart from any fair dealing for scholarly purposes as permitted under the Copyright Act, no part of this JOURNAL may be reproduced by any process without written permission from the Treasurer of the Australian Mathematical Society. 
In this paper we show that Jarratt's result can be generalized in a natural way: for all $\nu>0$, there are multipoint iterative methods (without memory) which use one function evaluation and $\nu$ derivative evaluations per iteration, and have order $2 \nu$. Jarratt's methods is an example with $\nu=2$, but the methods with $\nu>2$ appear to be new. Our sixth-order methods $(\nu=3)$ are more efficient than the fifth-order method of Jairatit [14].

Jarratt's results can also be extended to methods using higher derivatives. Our main result (Theorem 4.1 ) is that, for all $m>0, n \geqq 0$ and $k$ satisfying $m+1 \geqq k>0$, there are methods of order $m+2 n+1$ which use, for each iteration, one evaluation of $f, f^{\prime}, \cdots, f^{(m)}$ (at the same point), followed by $n$ evaluations of $f^{(k)}$ (at distinct points). These methods are described in Section 2, and the order of convergence is established in Section 4. The theoretical efficiencies of the different methods are compared in Section 5.

Special cases of practical interest $(k \leqq 3, n \leqq 3)$ are given explicitly in Section 6. Fortran subprograms for the methods of order four, six and eight (with $k=m=1$ and $n=1,2$ and 3) are given in Brent [5], [6]. Numerical results for these methods are summarized in Section 9, and some possible extensions are mentioned in Section 7.

Since our methods are useful for functions whose derivatives can be evaluated easily, it is not surprising that they are related to certain Runge-Kutta methods for solving a restricted class of ordinary differential equations. This is discussed in Section 8, and numerical comparisons with well-known RungeKutta methods are included in Section 9.

The theory of most of our zero-finding methods depends on the theory of orthogonal and "almost orthogonal" polynomials. We assume several wellknown properties of orthogonal polynomials, but some nonstandard results which we need later are proved in Section 3. These results, which are related to those of Micchelli and Rivlin [24], may be of independent interest.

\section{The methods}

Let $k, m$ and $n$ be integers satisfying $m>0, n \geqq 0, m+1 \geqq k>0$, and let $f$ be a sufficiently smooth function of one real variable with a simple zero $\zeta$. (It is sufficient for $f$ to have a continuous $(m+2 n+1)$-th derivative in a neighbourhood of $\zeta$.) We describe two classes of methods for improving an initial approximation $x_{0}$ to $\zeta$, using evaluations of $f\left(x_{0}\right), f^{\prime}\left(x_{0}\right), \cdots, f^{(m)}\left(x_{0}\right)$ and $\left.f^{(k)} y_{1}\right)$, $\cdots, f^{(k)}\left(y_{n}\right)$, where the points $y_{1}, \cdots, y_{n}$ will be specified below. After generating an improved approximation $x_{1}, x_{0}$ may be replaced by $x_{1}$ and a new approximation $x_{2}$ generated in the same way, etc. Since all the methods considered are stationary and without memory, it is sufficient to describe how $x_{1}$ is generated from $x_{0:}$ 
Methods in the first class, $B(k, m, n)$, have order $\min (m+2 n+1,2 m+n+1)$. The second class, $C(k, m, n)$, is a modification of $B(k, m, n)$, and metods in $C(k, m, n)$ have order $m+2 n+1$.

For our purposes it is sufficient to say that a method has order of convergence $\rho>1$ if $\rho$ is the greatest number such that $x_{1}-\zeta=O\left(\left|x_{0}-\zeta\right|^{p}\right)$ for all starting values $x_{0}$ sufficiently close to the simple zero $\zeta$. (More general definitions are given in Brent [4], [6] and Ortega and Rheinboldt [26].) The order is an integer for all methods considered below.

For $p+1>q>0$, the Jacobi polynomial $G_{n}(p, q, x)$ is the monic polynomial, of degree $n$, which is orthogonal to all polynomials of degree less than $n$ with respect to the weight function $(1-x)^{p-a} x^{q-1}$ on the interval $[0,1]$. (Our notation follows that of Abramowitz and Stegun [1].) Thus $G_{0}(p, q, x)=1$, $G_{1}(p, q, x)=x-q /(p+1), G_{2}(p, q, x)=x^{2}-2(q+1) x /(p+3)+q(q+1) /$ $((p+2)(p+3))$, etc.

\section{The class $B(k, m, n)$}

We say that a method belongs to $B(k, m, n)$ if an iteration generates a new approximation $x_{1}$ to $\zeta$, from an old approximation $x_{0}$, in the following way:

1. Evaluate $f_{0}^{(i)}=f^{(i)}\left(x_{0}\right)$ for $i=0,1, \cdots, m$.

2. If $f_{0}^{(0)}=0$ set $x_{1}=x_{0}$ and stop, otherwise set $\delta=\left|f_{0}^{(0)} / f_{0}^{(1)}\right|$.

3 . Let $z_{1}$ be an approximate zero of the polynomial

$$
p_{1}(x)=\sum_{i=0}^{m}\left(x-x_{0}\right)^{i} f_{0}^{(i)} / i !,
$$

satisfying the conditions

$$
z_{1}=x_{0}+O(\delta)
$$

and

$$
p_{1}\left(z_{1}\right)=O\left(\delta^{m+1}\right) .
$$

4. Evaluate $f_{i}^{(k)}=f^{(k)}\left(y_{i}\right)$, where $y_{i}=x_{0}+\alpha_{i}\left(z_{1}-x_{0}\right)$, for $i=1, \cdots, n$. Here $\alpha_{1}, \cdots, \alpha_{n}$ denote the zeros of $G_{n}(m+1, m+2-k, x)$ in some fixed order.

5. Let $p_{n+1}$ be the polynomial of degree at most $m+n$, satisfying $p_{n+1}^{(i)}\left(x_{0}\right)=f_{0}^{(i)}$ for $i=0, \cdots, m$ and $p_{n+1}^{(k)}\left(y_{i}\right)=f_{i}^{(k)}$ for $i=1, \cdots, n$. Let $x_{1}$ be an approximate zero of $p_{n+1}$, satisfying

$$
x_{1}=x_{0}+O(\delta)
$$

and

$$
p_{n+1}\left(x_{1}\right)=O\left(\delta^{p}\right)
$$

where $\rho=\min (m+2 n+1,2 m+n+1)$. 


\section{Comments on $B(k, m, n)$}

We do not specify how $z_{1}$ is computed so long as (2.1) and (2.2) hold. One method is to perform $\left[\log _{2}(m+1)\right]-1$ iterations of Newton's method, starting from the approximate zero $x_{0}-f_{0}^{(0)} / f_{0}^{(1)}$. Similar remarks apply for the computation of $x_{1}$.

$p_{1}(x)$ is the Taylor polynomial agreeing with $f\left(x_{0}\right), \cdots, f^{(i i)}\left(x_{0}\right)$. Conditions (2.1) and (2.3) ensure that $z_{1}$ and $x_{1}$ are approximations to the correct zeros of $p_{1}$ and $p_{n+1}$ respectively. If $x_{0}$ is sufficiently close to $\zeta$ then Newton's method gives the approximation $\tilde{x}_{0}=x_{0}-f_{0}^{(0)} / f_{0}^{(1)}$ satisfying $\tilde{x}_{0}=\zeta+O\left(\left(x_{0}-\zeta\right)^{2}\right)$, but $\left|\tilde{x}_{0}-x_{0}\right|=\delta$, so we may assume that

$$
\delta / 2 \leqq\left|x_{0}-\zeta\right| \leqq 2 \delta .
$$

Thus, any terms $O\left(\delta^{i}\right)$ are equivalent to terms $O\left(\left|x_{0}-\zeta\right|^{i}\right)$.

Since $\zeta$ is a simple zero of $f$, (2.2) is equivalent to $z_{1}=\zeta_{1}+O\left(\delta^{m+1}\right)$, where $\zeta_{1}$ is the zero of $p_{1}$ closest to $\zeta$. By the theory of Hermite interpolation (Traub [32]), $\zeta_{1}=\zeta+O\left(\delta^{m+1}\right)$, so (2.2) is equivalent to

$$
z_{1}=\zeta+O\left(\delta^{m+1}\right) .
$$

Theorem 2.1. If $M$ is in $B(k, m, n)$ and $x_{0}$ is sufficiently close to a simple zero $\zeta$, then steps 1 to 5 of $M$ are well-defined, and

$$
x_{1}=\zeta+O\left(\left|x_{0}-\zeta\right|^{\rho}\right)
$$

where

$$
\rho=\min (m+2 n+1,2 m+n+1) .
$$

SKetch of Proof. We shall not give the proof of Theorem 2.1 in detail, since it is similar to (but easier than) the proof of Theorem 4.1. We shall, however, indicate how the order $\rho$ given by (2.8) arises.

From the definition of $p_{n+1}$ (step 5 above) and the Taylor series expansion of $f$ about $x_{0}$, it is easy to show that

$$
p_{n+1}(x)=f(x)+O\left(\delta^{m+n+1}\right)
$$

and

$$
p_{n^{+}}^{\prime}(x)=f^{\prime}(x)+O\left(\delta^{m+n}\right)
$$

for all $x$ in the region of interest (say $\left[x_{0}-4 \delta, x_{0}+4 \delta\right]$ in view of (2.5)). Using properties of the Jacobi polynomial $G_{n}(m+1, m+2-k, x)$, as in Lemma 4.3 below, there is a kind of "superconvergence" phenomenon (de Boor and Swartz [2], Osborne [28]) at $z_{1}$ : 


$$
p_{n+1}\left(z_{1}\right)=f\left(z_{1}\right)+O\left(\delta^{m+2 n+1}\right)
$$

in contrast to (2.9).

Let $\bar{x}_{1}$ be the exact zero of $p_{n+1}$ near $x_{1}$. Using (2.6), (2.9) and (2.10), we have

$$
\left|\tilde{x}_{1}-z_{1}\right| \leqq\left|\tilde{x}_{1}-\zeta\right|+\left|z_{1}-\zeta\right|=O\left(\delta^{m+1}\right) .
$$

Now

$$
\begin{aligned}
\left|f\left(\tilde{x}_{1}\right)\right| & =\left|p_{n+1}\left(\tilde{x}_{1}\right)-f\left(\tilde{x}_{1}\right)\right| \\
& \leqq\left|p_{n+1}\left(z_{1}\right)-f\left(z_{1}\right)\right|+\left|p_{n+1}^{\prime}(\xi)-f^{\prime}(\xi)\right| \cdot\left|\tilde{x}_{1}-z_{1}\right|
\end{aligned}
$$

for some $\xi$ between $\tilde{x}_{1}$ and $z_{1}$. Using (2.10), (2.11) and (2.12), this gives

$$
f\left(\bar{x}_{1}\right)=O\left(\delta^{m+2 n+1}\right)+O\left(\delta^{2 m+n+1}\right)=O\left(\delta^{p}\right),
$$

and thus (as $f^{\prime}(x)$ is bounded away from zero near $\zeta$ )

$$
\tilde{x}_{1}=\zeta+O\left(\delta^{p}\right) \text {. }
$$

Since (2.3) and (2.4) ensure that $x_{1}=\tilde{x}_{1}+O\left(\delta^{\rho}\right)$, the result (2.7) follows.

\section{The class $C(k, m, n)$}

Methods in the class $B(k, m, n)$ are unsatisfactory if $m<n$, since their order is $2 m+n+1$, less than the order $m+2 n+1$ which might be expected from (2.11). The higher order is attainable if $z_{1}$ is updated and the zeros $\alpha_{1}, \cdots, \alpha_{n}$ are perturbed suitably after each evaluation of $f^{(k)}\left(y_{i}\right)$, so the second term in (2.13) is reduced to $O\left(\delta^{m+2 n+1}\right)$ or less, without substantially increasing the first term. We say that a method belongs to $C(k, m, n)$ if an iteration generates a new approximation $x_{1}$ to $\zeta$, from an old approximation $x_{0}$, in the following way:

1. Evaluate $f_{0}^{(i)}=f^{(i)}\left(x_{0}\right)$ for $i=0,1, \cdots, m$.

2. If $f_{0}^{(0)}=0$ set $x_{1}=x_{0}$ and stop, otherwise set $\delta=\left|f_{0}^{(0)} / f_{0}^{(1)}\right|$.

3. For $i=1,2, \cdots, n$ do steps 4 to 7 .

4. Let $p$, be the polynomial of degree at most $m+i-1$, satisfying $p_{i}^{(j)}\left(x_{0}\right)=f_{0}^{(i)}$ for $j=0,1, \cdots, m$ and $p_{i}^{(k)}\left(y_{j}\right)=f_{i}^{(k)}$ for $j=1, \cdots, i-1$. Let $z_{i}$ be an approximate zero of $p_{i}$, satisfying the conditions

$$
z_{i}=x_{0}+O(\delta)
$$

and

$$
p_{i}\left(z_{i}\right)=O\left(\delta^{m+i}\right)
$$


5. If $i>1$, compute

$$
\alpha_{i, j}=\alpha_{i-1, j}\left(z_{1-1}-x_{0}\right) /\left(z_{i}-x_{0}\right)
$$

for $j=1, \cdots, i-1$.

6. Let $q$, be the monic polynomial of degree $n+1-i$, satisfying

$$
\int_{0}^{1} P(x) q_{1}(x) x^{m+i-i}(1-x)^{i-i}\left(\prod_{j=1}^{i-1}\left(x-\alpha_{1, j}\right)\right) d x=\hat{0}
$$

for all polynomials $P$ of degree $n-i$. Let $\alpha_{i, i}$ be an approximate zero of $q_{i}$, satisfying

$$
\alpha_{l, i}=\alpha_{i}+O(\delta)
$$

and

$$
q_{t}\left(\alpha_{t, 1}\right)=O\left(\delta^{m+i-1}\right) .
$$

7. Evaluate $f_{1}^{(k)}=f^{(k)}\left(y_{i}\right)$, where

$$
y_{1}=x_{0}+\alpha_{i, s}\left(z_{i}-x_{0}\right) .
$$

8. Let $p_{n+1}$ be the polynomial, of degree at most $m+n$, satisfying $p_{n+1}^{(1)}\left(x_{0}\right)=$ $f_{0}^{(i)}$ for $i=0, \cdots, m$ and $p_{n+1}^{(k)}\left(y_{i}\right)=f_{1}^{(k)}$ for $i=1, \cdots, n$. Let $x_{1}$ be an approximate zero of $p_{n+1}$, satisfying (2.3) and

$$
p_{n+1}\left(x_{1}\right)=O\left(\delta^{m+2 n+1}\right) .
$$

\section{Comments on $C(k, m, n)$}

It is easy to see that the class $C(k, m, n)$ is the same as $B(k, m, n)$ if and only if $n=0$ or 1 . Using $\left[\log _{2}(m+1)\right]-1$ iterations, $z_{i}$ could be computed by Newton's method from the approximation $x_{0}-f_{0}^{(0)} / f_{0}^{(1)}$ if $i=1$, and one iteration from the approximation $z_{i-1}$ if $i>1$. Similarly for $x_{1}$.

The existence and uniqueness of $q_{1}$ (for $x_{0}$ sufficiently close to $\zeta$ ) is shown constructively below. In the cases of practical interest, explicit formulae can be given for $\alpha_{\imath, i}$, so that there is no need to construct $q_{i}$ (see (6.5), (6.9), and (6.10) for some examples).

From (2.16) and (2.20),

$$
y_{i}=x_{0}+\alpha_{1, i}\left(z_{i}-x_{0}\right)
$$

for $j=i, i+1, \cdots, n$, so the effect of replacing the approximation $z_{i}$ to $\zeta$ by the approximation $z_{j}$ is the same as if we had used a slightly perturbed node $\alpha_{j, i}$ in place of $\alpha_{i, i}$. 
Before proving that a method in $C(k, m, n)$ has order $m+2 n+1$ (Theorem $4.1)$, we need some results on orthogonal polynomials. The next two sections may be omitted without loss of continuity.

\section{Some results on orthogonal polynomials}

Theorem 3.1 is a generalization of the well-known results that zeros of polynomials orthogonal with respect to a positive weight function interlace, and that the matrix $\boldsymbol{A}$ given by (3.1) is nonsingular (by unisolvency, this is true for any distinct $\alpha_{1}, \cdots, \alpha_{n}$, not necessarily zeros of $P_{n}$ ).

TheOREM 3.1. Let $P_{0}, \cdots, P_{n}$ be polynomials of degree $0, \cdots, n$, orthogonal with respect to the weight function $w(x)$ on $[a, b]$. If $w(x)$ is positive a.e. on $[a, b]$, and $\alpha_{1}, \cdots, \alpha_{n}$ are the zeros of $P_{n}$ in any order, then all leading principal minors of

$$
\boldsymbol{A}=\left[\begin{array}{ccc}
P_{n-1}\left(\alpha_{1}\right) & \cdots & P_{n-1}\left(\alpha_{n}\right) \\
\vdots & & \vdots \\
P_{0}\left(\alpha_{1}\right) & \cdots & P_{0}\left(\alpha_{n^{c}}\right)
\end{array}\right]
$$

are nonsingular.

Proof. Since $w$ is positive, there is a three-term recurrence relation of the form

$$
P_{l}(x)=\left(A_{j} x+B_{j}\right) P_{j+1}(x)+C_{j} P_{j+2}(x)
$$

for $j=0,1, \cdots$, and $A_{j} \neq 0$ (see Isaacson and Keller ([12], Ch. 5) or Szegö ([31], Thm. 3.2.1)). Let $x$ be a zero of $P_{n}$. Applying (3.2) with $j=n-2$, $n-3, \cdots, n-p-1$ gives

$$
P_{n-p-1}(x)=\phi_{n, p}(x) P_{n-1}(x)
$$

for $p=0,1, \cdots, n-1$, where $\phi_{n, p}(x)$ is a polynomial of degree $p$ in $x$, with leading term $A_{n-2} \cdots A_{n-p-1} x^{p}$.

Suppose $0<s \leqq n$, and let $\boldsymbol{A}_{s}$ be the leading principal minor of order $s$ of A. Using (3.3) with $x=\alpha_{1}, \cdots, \alpha_{s}$, we have

$$
\operatorname{det}\left(\boldsymbol{A}_{s}\right)=\left(\prod_{i=1}^{s} P_{n-1}\left(\alpha_{1}\right)\right) \operatorname{det}\left[\begin{array}{ccc}
\phi_{n, 0}\left(\alpha_{1}\right) & \cdots & \phi_{n, 0}\left(\alpha_{s}\right) \\
\vdots & & \vdots \\
\phi_{n, s-1}\left(\alpha_{1}\right) & \cdots & \phi_{n, s-1}\left(\alpha_{s}\right)
\end{array}\right] .
$$

By performing row operations and using the observation above on the leading term $\phi_{n, p}$, this gives

$$
\operatorname{det}\left(\boldsymbol{A}_{s}\right)=\left(\prod_{i=1}^{s} P_{n-1}\left(\alpha_{i}\right)\right)\left(\prod_{i=2}^{s} A_{n-i}^{s+1-i}\right) \operatorname{det}\left(V_{s}\right),
$$


where

$$
V_{s}=\left[\begin{array}{ccc}
1 & \cdots & 1 \\
\alpha_{1} & \cdots & \alpha_{s} \\
\vdots & & \vdots \\
\alpha_{1}^{s-1} & \cdots & \alpha_{s}^{s-1}
\end{array}\right]
$$

is a Vandermonde matrix. Since the $\alpha_{1}$ are distinct and not zeros of $P_{n-1}$, the result follows from (3.5).

The idea of the following theorem is most easily seen by considering the special case $\beta_{t}=\alpha_{1}(i=1, \cdots, n)$ first. Then $d_{0}=\cdots=d_{n-1}=0,(3.11)$ says that $\gamma_{1}, \cdots, \gamma_{s}$ are slight perturbations of $\alpha_{1}, \cdots, \alpha_{s}$, and the theorem states that there exist slight perturbations $\gamma_{s+1}, \cdots, \gamma_{n}$ of $\alpha_{s+1}, \cdots, \alpha_{n}$, such that $\Pi_{j=1}^{n}\left(x-\gamma_{1}\right)$ is exactly orthogonal to polynomials of degree less than $n-s$, and approximately orthogonal to polynomials of degree $n-s, \cdots, n-1$. We state the more complicated result (with $\beta_{1}, \cdots, \beta_{n}$ slight perturbations of $\alpha_{1}, \cdots, \alpha_{n}$ ) because in Section 4 we shall apply Theorem 3.2 several times, and the $\gamma_{1}, \cdots, \gamma_{n}$ of one application will be (close to) the $\beta_{1}, \cdots, \beta_{n}$ of the next application.

Theorem 3.2. Let $P_{0}, \cdots, P_{n}$ be orthonormal polynomials (of degree $0, \cdots, n)$ with respect to the positive weight function $w$ on $[a, b]$, and let $\alpha_{1}, \cdots, \alpha_{n}$ be the zeros of $P_{n}$ in some fixed order. Suppose $0<s<n, \delta$ sufficiently small, and $\beta_{1}, \cdots, \beta_{n}$ satisfy

$$
\left|\beta_{i}-\alpha_{i}\right| \leqq \delta
$$

for $i=1, \cdots, n$. Suppose that there is a positive constant $c_{1}$, and numbers $\delta_{1}, \cdots, \delta_{s}$, such that

$$
\begin{aligned}
& \delta \geqq \delta_{1} \geqq \cdots \geqq \delta_{s} \geqq 0,
\end{aligned}
$$

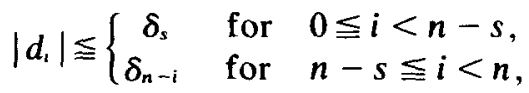

and

$$
d_{n}=1 \text {, }
$$

where

$$
d_{i}=c_{1} \int_{a}^{b} P_{1}(x)\left(\prod_{j=1}^{n}\left(x-\beta_{i}\right)\right) w(x) d x
$$


Finally, suppose $\gamma_{1}, \cdots, \gamma_{s}$ satisfy

$$
\left|\gamma_{i}-\beta_{i}\right| \leqq \delta_{s}
$$

for $i=1, \cdots, s$. Then there is a positive constant $c_{2}$ and unique $\gamma_{s+1}, \cdots, \gamma_{n}$ such that

$$
\gamma_{1}=\beta_{i}+O\left(\delta_{s}\right)
$$

for $i=s+1, \cdots, n$, and

$$
e_{i}=\left\{\begin{array}{ccc}
0 & \text { for } & 0 \leqq i<n-s, \\
O\left(\delta_{n-i}\right) & \text { for } & n-s \leqq i<n, \\
1 & \text { for } & i=n,
\end{array}\right\}
$$

where

$$
e_{i}=c_{2} \int_{a}^{b} P_{i}(x)\left(\prod_{i=1}^{n}\left(x-\gamma_{J}\right)\right) w(x) d x
$$

Proof. Let

$$
\gamma_{1}^{\prime}=\left\{\begin{array}{lll}
\gamma_{1} & \text { if } & i \leqq s \\
\beta_{i} & \text { if } & i>s
\end{array}\right\}, \quad q_{1}(x)=c_{1} \prod_{i=1}^{n}\left(x-\gamma_{i}^{\prime}\right)
$$

and $q_{2}(x)=q_{1}(x)+\sum_{t=0}^{n-1} \mu_{i} P_{t}(x)$, where the constants $\mu_{i}=O\left(\delta_{s}\right)$ will be determined below. From (3.11),

$$
q_{1}(x)=c_{1} \prod_{i=1}^{n}\left(x-\beta_{i}\right)+O\left(\delta_{s}\right) \text { for all } x \text { in }[a, b],
$$

so from (3.8) to (3.10) we have

$$
f_{i}=\left\{\begin{array}{ccc}
O\left(\delta_{s}\right) & \text { for } & 0 \leqq i<n-s \\
O\left(\delta_{n-i}\right) & \text { for } & n-s \leqq i<n \\
1+O\left(\delta_{s}\right) & \text { for } & i=n,
\end{array}\right\}
$$

where

$$
f_{i}=\int_{a}^{b} P_{i}(x) q_{1}(x) w(x) d x .
$$

Let

$$
g_{i}=\int_{a}^{b} P_{1}(x) q_{2}(x) w(x) d x .
$$

Since the $P_{i}$ are orthonormal, (3.16) and (3.17) give

$$
g_{i}=f_{i}+\mu_{i}
$$

for $i=0, \cdots, n-1$. Set $\mu_{i}=-f_{i}$ for $i=0, \cdots, n-s-1$. 
From Wilkinson ([33], Sec. 2.7), the zeros $\gamma_{1}^{\prime \prime}$ of $q_{2}$ are analytic functions of $\mu_{n-s}, \cdots, \mu_{n-1}$, given by

$$
\gamma_{i}^{\prime \prime}=\gamma_{i}^{\prime}+\sum_{j=0}^{n-s-1} f_{j} P_{j}\left(\gamma_{i}^{\prime}\right) / q_{1}^{\prime \prime}\left(\gamma_{i}^{\prime}\right)-\sum_{i=n-s}^{n-1} \mu_{j} P_{j}\left(\gamma_{i}^{\prime}\right) / q_{1}^{\prime}\left(\gamma_{i}^{\prime}\right)+O\left(\delta_{s}^{2}\right)
$$

$(i=1, \cdots, n)$, provided $\mu_{j}=O\left(\delta_{s}\right)$ for $j=n-s, \cdots, n-1$. By (3.7), (3.8), (3.11) and Theorem 3.1, the matrix

$$
\boldsymbol{A}=\left[\begin{array}{ccc}
P_{n-s}\left(\gamma_{1}^{\prime}\right) & \cdots & P_{n-s}\left(\gamma_{s}^{\prime}\right) \\
\vdots & & \vdots \\
P_{n-1}\left(\gamma_{1}^{\prime}\right) & \cdots & P_{n-1}\left(\gamma_{s}^{\prime}\right)
\end{array}\right]
$$

is nonsingular for $\delta$ sufficiently small, so there exist $\mu_{n-s}, \cdots, \mu_{n-1}$ (all $O\left(\delta_{s}\right)$ ) such that $\gamma_{1}^{\prime \prime}=\gamma_{1}^{\prime}$ for $i=1, \cdots, s$. Hence (by definition of $\gamma_{i}^{\prime}$ ), $\gamma_{1}=\gamma_{i}^{\prime \prime}$ for $i=1, \cdots, s$. Take $\gamma_{i}=\gamma_{i}^{\prime \prime}$ for $i=s+1, \cdots, n$ also, so

$$
q_{2}(x)=c_{1} \prod_{i=1}^{n}\left(x-\gamma_{i}\right) .
$$

By (3.19) and the construction of $\mu$, and $\gamma_{t}$, (3.12) holds. From (3.18) and the choice of $\mu_{0}, \cdots, \mu_{n-s-1}, g_{1}=0$ for $i<n-s$. From (3.8), (3.15) and (3.18), $g_{i}=O\left(\delta_{n-i}\right)$ for $n-s \leqq i<n$, and $g_{n}=1+O\left(\delta_{s}\right)$. Taking $c_{2}=c_{1} / g_{n}$ and collecting these results, existence follows. Uniqueness (subject to (3.12)) follows from (3.19) and the nonsingularity of $\boldsymbol{A}$.

The following corollary gives an extension to "almost orthogonal" polynomials of a classical result for orthogonal polynomials (the case $\delta=0, \gamma_{t}=\alpha_{t}$ ). The proof follows that of the classical result up to equation (3.23), and then uses Theorem 3.2 .

Corollary 3.1. Under the conditions of Theorem 3.2, there exist weights $w_{1}, \cdots, w_{n}$ such that

where

$$
\sum_{i=1}^{n} w_{i} \gamma_{i}^{j}=\int_{a}^{b} x^{j} w(x) d x+r_{i}
$$

$$
r_{j}=\left\{\begin{array}{cc}
0 & \text { for } j=0,1, \cdots, 2 n-(s+1), \\
O\left(\delta_{2 n-1}\right) & \text { for } j=2 n-s, \cdots, 2 n-1
\end{array}\right.
$$

Proof. We may assume $\delta$ sufficiently small that $\gamma_{1}, \cdots, \gamma_{n}$ are distinct (in view of (3.7), (3.11) and (3.12)). For any function $f$ we have the Lagrange interpolation formula

$$
f(x)=\sum_{i=1}^{n} L_{i}(x) f\left(\gamma_{i}\right)+\left(\prod_{i=1}^{n}\left(x-\gamma_{i}\right)\right) f\left[\gamma_{1}, \cdots, \gamma_{n}, x\right],
$$


where

$$
L_{1}(x)=\prod_{\substack{j=1 \\ i \neq i}}^{n}\left(\frac{x-\gamma_{j}}{\gamma_{1}-\gamma_{1}}\right)
$$

Let

$$
w_{\imath}=\int_{a}^{b} L_{\imath}(x) w(x) d x,
$$

and integrate both sides of (3.22), with $f(x)=x^{j}$. Thus (3.21) holds if

$$
r_{i}=-\int_{a}^{b}\left(\prod_{i=1}^{n}\left(x-\gamma_{i}\right)\right) f\left[\gamma_{1}, \cdots, \gamma_{n}, x\right] w(x) d x .
$$

Since $f\left[\gamma_{1}, \cdots, \gamma_{n}, x\right]$ vanishes for $j<n$, and is a polynomial of degree $j-n$ for $j \geqq n$, the result follows by expanding this polynomial as a sum $\sum_{i=0}^{j-n} \nu_{i} P_{1}(x)$ (where $\nu_{1}=O(1)$ ) and applying Theorem 3.2.

\section{Order of convergence}

Before proving the main result (Theorem 4.1) we need some lemmas. The notation of Section 2 is assumed.

Lemma 4.1. If $M$ is in $C(k, m, n)$ and $x_{0}$ is sufficiently close to the simple zero $\zeta$, then $M$ is well-defined. If, in addition, $x_{0} \neq \zeta$, then (4.1) to (4.5) hold:

$$
\begin{aligned}
\delta / 2 & \leqq\left|x_{0}-\zeta\right| \leqq 2 \delta ; \\
z_{t} & =\zeta+O\left(\delta^{m+\imath}\right)
\end{aligned}
$$

for $i=1, \cdots, n ; q$, exists, is unique, and has a zero

$$
\alpha_{i, 4}^{\prime}=\alpha_{i}+O(\delta)
$$

for $i=1, \cdots, n$;

$$
\alpha_{i, j}=\alpha_{i-1, i}+O\left(\delta^{m+i-2}\right)
$$

for $0<j<i \leqq n ;$ and

$$
x_{1}=\zeta+O\left(\delta^{m+n+1}\right)
$$

Proof. The exceptional case $x_{0}=\zeta$ is covered by step 2 of $M(k, m, n)$, so we may assume $x_{0} \neq \zeta$. The inequality (4.1) follows in the same way as (2.5).

(4.2) to (4.4) clearly hold for $i=1$. We shall assume that they hold for $i<t$ (where $1<t \leqq n$ ) and prove that they hold for $i=t$.

$p_{t}$ is a well-defined polynomial of degree $m+t-1$, and the theory of polynomial interpolation (Traub [32]) shows that there is a zero 
$\zeta_{t}=\zeta+O\left(\delta^{m+t}\right)$ of $p_{t}$. Conditions (2.14) and (2.15) ensure that $z_{t}=\zeta_{t}+O\left(\delta^{m+1}\right)$, so (4.2) with $i=t$ follows.

From (2.16),

$$
\alpha_{t, j}=\alpha_{t-1, j}\left(1+\frac{z_{t-1}-z_{t}}{z_{t}-x_{0}}\right)
$$

for $0<i<t$. Since

$$
\left|z_{t-1}-z_{t}\right| \leqq\left|z_{t-1}-\zeta\right|+\left|z_{t}-\zeta\right|=O\left(\delta^{m+t-1}\right)
$$

and (using (4.1))

$$
\begin{aligned}
\left|z_{\imath}-x_{0}\right| & \geqq\left|x_{0}-\zeta\right|-\left|z_{\imath}-\zeta\right| \\
& \geqq \delta / 2-O\left(\delta^{m+t}\right) \geqq \delta / 4
\end{aligned}
$$

for $\delta$ sufficiently small, (4.4) with $i=t$ follows.

From (4.4) with $i=t, t-1, \cdots, j+1$, we have $\alpha_{t, j}=\alpha_{i, j}+O\left(\delta^{m+i-1}\right)$, but (by the inductive hypothesis) $\alpha_{t,}=\alpha_{j}+O(\delta)$, so $\alpha_{t, j}=\alpha_{j}+O(\delta)$ for $j=1, \cdots, t-1$. Thus, Theorem 3.2 shows that $q_{1}$ exists, is unique, and has a zero $\alpha_{t, t}^{\prime}=$ $\alpha_{t}+O(\delta)$ which $\alpha_{t, t}$ approximates. This completes the proof of (4.2) to (4.4), by induction on $i$. Finally, (4.5) follows in the same way as (4.2).

Lemma 4.2. If a method in $C(k, m, n)$ is applied with $x_{0}$ sufficiently close to $\zeta$, but $x_{0} \neq \zeta$, then there exist weights $w_{1}, \cdots, w_{n}$ (all $\left.O(1)\right)$ such that

$$
\sum_{i=1}^{n} w_{i} \alpha_{n, 1}^{\prime}=\frac{(j+m+1-k) !(k-1) !}{(j+m+1) !}+O\left(\delta^{2 n-j}\right)
$$

for $j=0,1, \cdots, 2 n-1$.

Proof. The first time step 6 is performed, we have $i=1, q_{1}(x)=$ $G_{n}(m+1, m+2-k, x)$ and, from (2.18) and (2.19), $\alpha_{1,1}=\alpha_{1}+O\left(\delta^{m}\right)$. For subsequent executions of step 6 we have $i>1$ and, from (2.22) and Lemma 4.1, it is easy to show (by induction on $i$ ) that Theorem 3.2 is applicable (with $s=i-1, \delta_{i}=O\left(\delta^{m+i-1}\right)$ for $j=1, \cdots, i-1, w(x)=(1-x)^{k-1} x^{m+1-k}$, etc. $)$. After the $n$-th execution of step 6, Corollary 3.1 (with $s=n-1$ ) shows that there exist weights $w_{1}$ such that

$$
\begin{aligned}
\sum_{i=1}^{n} w_{i} \alpha_{n, i}^{i}- & \int_{0}^{1} x^{i+m+1-k}(1-x)^{k-1} d x \\
& =\left\{\begin{array}{ccc}
0 & \text { if } 0 \leqq j \leqq n, \\
O\left(\delta^{m+2 n-j-1}\right) & \text { if } & n<j<2 n .
\end{array}\right\}
\end{aligned}
$$

Since $m+2 n-j-1 \geqq 2 n-j$, and the integral on the left side of (4.7) is equal to $(j+m+1-k) !(k-1) ! /(j+m+1)$ !, the result follows. (In fact (4.7) is stronger than (4.6), but (4.6) is sufficient for later applications.) 
Lemma 4.3. Suppose $K$ constant, $P(x)$ a polynomial, of degree at most $m+2 n$, with coefficients bounded by $K$, satisfying $P(0)=\cdots=P^{(m)}(0)=0$ and $P^{(k)}\left(\beta_{1} \varepsilon\right)=\cdots=P^{(k)}\left(\beta_{n} \varepsilon\right)=0$, where

$$
\sum_{i=1}^{n} w_{i} \beta_{i}^{j}=(j+m+1-k) !(k-1) ! /(j+m+1) !+O\left(\varepsilon^{2 n-j}\right)
$$

for $j=0,1, \cdots, 2 n-1$. Then $P(\varepsilon)=O\left(\varepsilon^{m+2 n+1}\right)$.

Proof. Let

$$
\begin{gathered}
P(x)=\sum_{i=0}^{2 n-1} a_{1} x^{j+m+1} \text {, so } P^{(k)}\left(\beta_{1} \varepsilon\right)=0 \text { gives } \\
\sum_{j=0}^{2 n-1} a_{1} \frac{(j+m+1) !}{(j+m+1-k) !}\left(\beta_{l} \varepsilon\right)^{j+m+1-k}=0 .
\end{gathered}
$$

Multiplying each side of (4.9) by $w_{i} \beta_{i}^{k-m-1} \varepsilon^{k} /(k-1)$ ! and summing over $i=1, \cdots, n$ gives

$$
\sum_{j=0}^{2 n-1}\left(a_{j} \varepsilon^{j+m+1} \sum_{i=1}^{n} \frac{w_{i} \beta^{!}(j+m+1) !}{(j+m+1-k) !(k-1) !}\right)=0 .
$$

Thus, the result follows from (4.8).

Lemma 4.4. If $\boldsymbol{n}>\mathbf{0}, x_{0}$ is sufficiently close to the simple zero $\zeta$ of $f$, and $M$ in $C(k, m, n)$ is applied, then (assuming $\left.x_{0} \neq \zeta\right)$

$$
f\left(z_{n}\right)=p_{n+1}\left(z_{n}\right)+O\left(\delta^{m+2 n+1}\right)
$$

and

$$
\sup _{\xi \in\left[x_{0}-4 \delta . \times x_{0}+4 \delta\right]}\left|f^{\prime}(\xi)-p_{n^{+}}^{\prime}(\xi)\right|=O\left(\delta^{m+n}\right) .
$$

Proof. Let $f(x)=f_{1}(x)+f_{2}(x)$, where

$$
f_{1}(x)=\sum_{j=0}^{m+2 n}\left(x-x_{0}\right)^{\prime} f^{(i)}\left(x_{0}\right) / j ! .
$$

For $i=1,2$, let $r_{i}(x)$ be the polynomial, of degree at most $m+n$, satisfying $r_{1}^{(j)}\left(x_{0}\right)=f_{i}^{(j)}\left(x_{0}\right)$ for $j=0, \cdots, m$ and $r_{i}^{(k)}\left(y_{j}\right)=f_{1}^{(k)}\left(y_{1}\right)$ for $j=1, \cdots, n$, where $y_{1}$ is defined by (2.20). Thus, from the definition of $p_{n+1}$,

$$
p_{n+1}(x)=r_{1}(x)+r_{2}(x) .
$$

Since $P(x)=f_{1}\left(x_{0}+x\right)-r_{1}\left(x_{0}+x\right)$ is a polynomial of degree at most $m+2 n$ in $x$, and the conditions of Lemma 4.3 are satisfied with $\varepsilon=z_{n}-x_{0}=$ $O(\delta), \beta_{i}=\alpha_{n, i}(i=1, \cdots, n)$, and $w_{1}$ given by Lemma 4.2 , we have $P(\varepsilon)=$ $O\left(\varepsilon^{m+2 n+1}\right)$, so 


$$
f_{1}\left(z_{n}\right)=r_{1}\left(z_{n}\right)+O\left(\delta^{m+2 n+1}\right) .
$$

We may write $r_{2}(x)=\sum_{j=0}^{m+n} a_{j}\left(x-x_{0}\right)^{j}$, and, from (4.12) and the definitions of $f_{2}$ and $r_{2}$, we have $a_{0}=\cdots=a_{m}=0$. The coefficients $a_{m+1}, \cdots, a_{m+n}$ are determined by the linear equations

$$
\sum_{j=m+1}^{m+n} \alpha_{n, i}^{i-k} \varepsilon^{i} u_{j} j ! /(j-k) !=\varepsilon^{k} f_{2}^{(k)}\left(y_{i}\right)
$$

for $i=1, \cdots, n$. From (4.12) and the definition of $f_{2}$, the right hand side of (4.15) is $O\left(\delta^{m+2 n+1}\right)$, and $\alpha_{n, 1}, \cdots, \alpha_{n, n}$ are distinct, so $\varepsilon^{\prime} a_{j}=O\left(\delta^{m+2 n+1}\right)$, for $j=$ $m+1, \cdots, m+n$. Thus $r_{2}\left(z_{n}\right)=O\left(\delta^{m+2 n+1}\right)$, and from (4.14) we have

$$
\begin{aligned}
\left|f\left(z_{n}\right)-p_{n+1}\left(z_{n}\right)\right| & \leqq\left|f_{1}\left(z_{n}\right)-r_{1}\left(z_{n}\right)\right|+\left|r_{2}\left(z_{n}\right)\right|+\left|f_{2}\left(z_{n}\right)\right| \\
& =O\left(\delta^{m+2 n+1}\right),
\end{aligned}
$$

so (4.10) is established. The proof of (4.11) is straightforward, and does not use the special properties of $\alpha_{n, 1}, \cdots, \alpha_{n, n}$ (except for their being distinct), so is omitted.

Using Lemmas 4.1 and 4.4 it is easy to prove our main result:

THEOREM 4.1. If $x_{0}$ is sufficiently close to the simple zero $\zeta$, then a method in $C(k, m, n)$ is defined, and $x_{1}=\zeta+O\left(\left|x_{0}-\zeta\right|^{m+2 n+1}\right)$.

Proof. Suppose $n>0$, for otherwise the result follows from Lemma 4.1. From equations (4.2) and (4.5) of Lemma 4.1, we have

$$
x_{1}=z_{n}+O\left(\delta^{m+n}\right) \text {. }
$$

Now

$$
\begin{aligned}
& \left|f\left(x_{1}\right)\right| \leqq\left|p_{n+1}\left(x_{1}\right)\right|+\left|f\left(x_{1}\right)-p_{n+1}\left(x_{1}\right)\right| \\
& \leqq\left|p_{n+1}\left(x_{1}\right)\right|+\left|f\left(z_{n}\right)-p_{n+1}\left(z_{n}\right)\right| \\
& +\left|f^{\prime}(\xi)-p_{n+1}^{\prime}(\xi)\right| \cdot\left|x_{1}-z_{n}\right|
\end{aligned}
$$

for some $\xi$ between $x_{1}$ and $z_{n}$. From (2.21), Lemma 4.4 and (4.16), this gives $f\left(x_{1}\right)=O\left(\delta^{m+2 n+1}\right)$. Since $\zeta$ is a simple zero of $f$, we may suppose that $f^{\prime}(x)$ is bounded away from zero in the region of interest, so $x_{1}=\zeta+O\left(\delta^{m+2 n+1}\right)$. The result now follows from (4.1).

\section{Theoretical comparison of various methods}

If an iterative method of order $\rho>1$ requires $w$ units of work per iteration, its efficiency is

$$
E=\frac{\log \rho}{w}
$$


The motivation for this definition (and a more general definition) is given in Brent [4]. In this section we compare the efficiencies of methods in the classes $C(1,1, n)$ for $n=0,1, \cdots$. The extension to methods in $C(k, m, n)$ is straightforward.

Theorem 4.1 shows that a method $M_{n}$ in $C(1,1, n)$ has order at least $2(n+1)$, and we shall assume that the order is exactly $2(n+1)$ (this is usually true: see Section 6). If the work for one evaluation of $f(x)$ is $w(f)$ and the overhead for one iteration is $w_{0}(n)$, then the total work per iteration is

$$
w=w(f)+(n+1) w\left(f^{\prime}\right)+w_{0}(n),
$$

so (from (5.1)) the efficiency is

$$
E_{n}=\log [2(n+1)] /\left[w(f)+(n+1) w\left(f^{\prime}\right)+w_{0}(n)\right] .
$$

We expect $w_{0}(n)$ to be an increasing function of $n$, and it can be estimated for any particular implementation of $M_{n}$. For the sake of simplicity, we shall assume $w_{0}(n)=0$ below. This is a reasonable approximation if $n$ is small and $f$ is difficult to evaluate (see also Kung and Traub [21], [22]).

With our simplifying assumption, (5.2) gives

$$
E_{n} / E_{0}=(1+r)\left(1+\log _{2}(n+1)\right) /(n+1+r),
$$

where $r=w(f) / w\left(f^{\prime}\right)$ and $E_{0}$ is the efficiency of Newton's method. Some values of $E_{n} / E_{0}$ are given in Table 5.1.

TABLE 5.1

$E_{n} / E_{0}$ for various $n$ and $r=w(f) / w\left(f^{\prime}\right)$

\begin{tabular}{rccccc}
\hline$r n$ & & & & & \\
& 1 & 2 & 3 & & 5 \\
\hline & & & & & \\
\hline .0 & 1.000 & 0.862 & 0.750 & 0.664 & 0.597 \\
1.0 & 1.200 & 1.108 & 1.000 & 0.906 & 0.827 \\
2.0 & 1.333 & 1.292 & 1.200 & 1.107 & 1.024 \\
5.0 & 1.500 & 1.551 & 1.500 & 1.424 & 1.344 \\
10.0 & 1.714 & 1.939 & 2.000 & 1.993 & 1.955 \\
$\infty$ & 1.833 & 2.187 & 2.357 & 2.436 & 2.465 \\
\hline
\end{tabular}

From (5.3) it may be shown that $M_{n}$ is the optimal method (from $\left.M_{0}, M_{1}, \cdots\right)$ if

$$
\phi(n)<r<\phi(n+1),
$$


where

$$
\phi(n)=\frac{\log (2 n)}{\log (1+1 / n)}-n .
$$

Thus, the optimal values of $n$ are

$$
n=\left\{\begin{array}{l}
1 \text { if } 0<r<1.419, \\
2 \text { if } 1.419<r<3.228, \\
3 \text { if } 3.228<r<5.319 \\
4 \text { if } 5.319<r<7.629, \\
5 \text { if } 7.629<r<10.120, \text { etc. }
\end{array}\right.
$$

In particular, Jarratt's method $(n=1)$ is always more efficient than Newton's method $(n=0)$, but it is less efficient than one of our sixth-order methods $(n=2)$ if $w(f)>1.419 w\left(f^{\prime}\right)$, etc.

It is interesting to compare our methods with methods which use only function evaluations. There are multipoint methods without memory which use $\nu+1$ function evaluations per iteration, and have order $2^{\nu}$. This order is known to be optimal for $\nu=1$ (Kung and Traub [20], [22]) and $\nu=2$ (Wozniakowski [36]), and is conjectured to be optimal for all $\nu \geqq 1$. Care has to be taken in phrasing the conjecture to avoid Winograd's encoding trick: one way is to suitably restrict the class of allowable iteration functions (see Brent [6]). Brent, Winograd and Wolfe [8] have shown that the optimal order is $2^{\nu+1}$ if memory is permitted. In contrast to these results, an obvious conjecture is that methods (without memory) which use one function evaluation and $\nu$ derivative evaluations per iteration have order at most $2 \nu$. Kung and Traub [22] proved this for $\nu=1$, and it has recently been proved for all $\nu \geqq 1$. In fact, Wozniakowski [37] has shown that the methods in $C(k, m, n)$ have optimal order in a wide class of methods (without memory) using the same information about $f$ at each iteration. This result was obtained independently by Meersman [39].

Our methods are only of practical interest for small $\nu$ (say $\nu=n+1 \leqq 4$ ), and some such methods are described in detail in the next section. Related methods with memory are given by King [17], [18].

\section{Some methods of practical interest}

In this section we use the notation of Section 2 as far as possible, and temporary variables used in the description below are denoted $\Delta_{i}, t_{i}, v_{i}, a, b, c$, etc. Specific methods in $C(k, m, n)$ (or in $B(k, m, n)$ if $m \geqq n$ ) are referred to as "method kmna", "method kmnb", etc. If a method has order $\rho$, the asymptotic error constant (if it exists) is

$$
K=\lim _{x_{0} \rightarrow \zeta}\left(x_{1}-\zeta\right) /\left(x_{0}-\zeta\right)^{\rho} .
$$


(It is usual to put absolute value signs in (6.1), but we omit them since $\rho$ is an integer for all the methods considered below.) Asymptotic error constants may be obtained, in terms of $f^{\prime}(\zeta), f^{\prime \prime}(\zeta)$, etc., by substituting the Taylor series expansion of $f(x)$ about $\zeta$ in the definition of the method, as described in Brent [6]. The error constants for the methods considered below can be expressed as sums of products of the form $c \prod_{i=2}^{p} \phi_{i}^{r}$, where (from Traub [32])

$$
\sum_{i=2}^{\rho}(i-1) r_{i}=\rho-1
$$

and

$$
\phi_{i}=\frac{f^{(i)}(\zeta)}{i ! f^{\prime}(\zeta)}
$$

\section{Fourth-order methods}

If $m=m=1$ and $k=1$ or 2 , the relevant Jacobi polynomial is $G_{1}(2,3-k, x)=x-(1-k / 3)$, so

$$
\alpha_{1}=1-k / 3 \text {. }
$$

Some fourth-order methods are summarized in Table 6.1. In all cases $\alpha_{1}$ is given by (6.4), $\Delta_{1}=-f_{0}^{(0)} / f_{0}^{(1)}$, and $f_{1}^{(k)}=f^{(k)}\left(x_{0}+\alpha_{1} \Delta_{1}\right)$. In some cases the auxiliary variable

$$
\Delta_{2}=\left\{\begin{array}{ccc}
3\left(f_{1}^{(1)}-f_{0}^{(1)}\right) /\left(6 f_{1}^{(1)}-2 f_{0}^{(1)}\right) & \text { if } & k=1 \\
\Delta_{1} f_{1}^{(2)} /\left[2\left(f_{0}^{(1)}+\Delta_{1} f_{1}^{(2)}\right)\right] & \text { if } & k=2
\end{array}\right.
$$

is used. The formulae for $x_{1}$ and the asymptotic error constants $K$ are given in the table. The only difference between the methods with $k=1$ is in the approximation used for the zero of the interpolating quadratic

$$
p_{2}(x)=f_{0}^{(0)}+f_{0}^{(1)}\left(x-x_{0}\right)+\frac{3\left(f_{1}^{(1)}-f_{0}^{(1)}\right)}{4 \Delta_{1}}\left(x-x_{0}\right)^{2} .
$$

Method 111a is Jarratt's method [13], method 111b uses the approximation $\tilde{x}_{0}-p_{2}\left(\tilde{x}_{0}\right) / p_{2}^{\prime}\left(\tilde{x}_{0}\right)$ where $\tilde{x}_{0}=x_{0}+\Delta_{1}$, method $111 \mathrm{c}$ uses the (better) approximation $\bar{x}_{0}-p_{2}\left(\tilde{x}_{0}\right) / p_{2}^{\prime}\left(\tilde{x}_{0}\right)-\frac{1}{2} p_{2}^{2}\left(\tilde{x}_{0}\right) p_{2}^{\prime \prime}\left(\tilde{x}_{0}\right) /\left(p_{2}^{\prime}\left(\tilde{x}_{0}\right)\right)^{3}$, and method 111d solves the quadratic exactly if it has real roots. Similarly, the difference between the methods with $k=2$ is in the approximation used for the zero of the interpolating quadratic $f_{0}^{(0)}+f_{0}^{(1)}\left(x-x_{0}\right)+\frac{1}{2} f_{1}^{(2)}\left(x-x_{0}\right)^{2}$. 
TABLE 6.1

Some fourth-order methods

Method $k \quad \alpha_{1}$

$x_{1}-x_{0}$

K

$\begin{array}{lll}111 \mathrm{a} & 1 & 2 / 3 \\ 111 \mathrm{~b} & 1 & 2 / 3 \\ 111 \mathrm{c} & 1 & 2 / 3 \\ 111 \mathrm{~d} & 1 & 2 / 3 \\ 211 \mathrm{a} & 2 & 1 / 3 \\ 211 \mathrm{~b} & 2 & 1 / 3\end{array}$

$$
\begin{gathered}
\Delta_{1}\left(5+3\left(f_{0}^{(1)} / f_{1}^{(1)}\right)^{2}\right) / 8 \\
\Delta_{1}\left(1-\Delta_{2}\right) \\
\Delta_{1}\left(1-\Delta_{2}\left(1+\Delta_{2}^{2}\right)\right) \\
2 \Delta_{1} /\left\{1+\left[\max \left(0,3 f_{1}^{(1)} / f_{0}^{(1)}-2\right)\right]^{\frac{1}{2}}\right\} \\
\Delta_{1}\left(1-\Delta_{2}\right) \\
\Delta_{1}\left(1-\Delta_{2}\left(1+\Delta_{2}^{2}\right)\right)
\end{gathered}
$$

$$
\begin{gathered}
\phi_{4} / 9-\phi_{2} \phi_{3}+13 \phi_{2}^{3} / 9 \\
\phi_{4} / 9-\phi_{2} \phi_{3}+\phi_{2}^{3} \\
\phi_{4} / 9-\phi_{2} \phi_{3} \\
\phi_{4} / 9-\phi_{2} \phi_{3} \\
\phi_{4} / 3-\phi_{2} \phi_{3}+\phi_{2}^{3} \\
\phi_{4} / 3-\phi_{2} \phi_{3}
\end{gathered}
$$

\section{Sixth-order methods}

If $k=m=1, n=2$, the relevant Jacobi polynomial is $G_{2}(2,2, x)=$ $x^{2}-6 x / 5+3 / 10$, with zeros $(6 \pm \sqrt{6}) / 10$.

Method 112a.

$$
\begin{gathered}
\alpha_{1}=(6-\sqrt{6}) / 10, \Delta_{1}=-f_{0}^{(0)} / f_{0}^{(1)}, f_{1}^{(1)}=f^{\prime}\left(x_{0}+\alpha_{1} \Delta_{1}\right), \\
\Delta_{2}=\frac{1}{2} \Delta_{1}\left(f_{1}^{(1)}+\left(2 \alpha_{1}-1\right) f_{0}^{(1)}\right) /\left(f_{1}^{(1)}+\left(\alpha_{1}-1\right) f_{0}^{(1)}\right), \alpha_{2,1}=\alpha_{1} \Delta_{1} / \Delta_{2}, \\
\alpha_{2,2}=\left(3-4 \alpha_{2,1}\right) /\left(4-6 \alpha_{2,1}\right), \\
f_{2}^{(1)}=f^{\prime}\left(x_{0}+\alpha_{2,2} \Delta_{2}\right), \\
t_{1}=\left(f_{1}^{(1)}-f_{0}^{(1)}\right) /\left(\alpha_{1} \Delta_{1}\right), t_{2}=\left(f_{2}^{(1)}-f_{0}^{(1)}\right) /\left(\alpha_{2,2} \Delta_{2}\right), \\
v_{1}=\left(\alpha_{2,2} t_{1}-\alpha_{2,1} t_{2}\right) /\left(\alpha_{2,2}-\alpha_{2,1}\right), v_{2}=\left(t_{2}-t_{1}\right) /\left(\alpha_{2,2}-\alpha_{2,1}\right), \\
\Delta_{3}=f_{0}^{(0)}+f_{0}^{(1)} \Delta_{2}+\left(3 v_{1}+2 v_{2}\right) \Delta_{2}^{2} / 6, \\
\Delta_{4}=f_{0}^{(1)}+\Delta_{2}\left(v_{1}+v_{2}\right),
\end{gathered}
$$

and

$$
x_{1}=x_{0}+\Delta_{2}-\Delta_{3} / \Delta_{4}-\frac{1}{2} \Delta_{3}^{2} v_{1} / \Delta_{4}^{3} .
$$

The error constant is

$$
K=\phi_{6} / 100+\left(1-5 \alpha_{1}\right) \phi_{2} \phi_{s} / 10+\left(3 \alpha_{1}-2\right) \phi_{3} \phi_{4} / 5 .
$$

METHOD 112b. This method is the same as $112 \mathrm{a}$, except $\alpha_{1}=(6+\sqrt{6}) / 10$. The error constant is still given by (6.7). 


\section{Comments on methods $112 a$ and $112 b$}

Most of the equations above are obtained in a straightforward manner from the general description in Section 2. We should explain equation (6.5). From (2.17) we need a linear polynomial $q_{2}(x)$ such that $\int_{0}^{1} q_{2}(x) x\left(x-\alpha_{2,1}\right) d x=$ 0 , and it is easily verified that $q_{2}(x)=x-\left(3-4 \alpha_{2,1}\right) /\left(4-6 \alpha_{2,1}\right)$ is the required polynomial. $\alpha_{2,1} \neq 2 / 3$ if $x_{0}$ is sufficiently close to $\zeta$, so $\alpha_{2,2}$ is well-defined.

$\Delta_{3}$ and $\Delta_{4}$ are respectively the value of the interpolating polynomial $p_{3}(x)$ and its derivative $p_{3}^{\prime}(x)$ at $x=z_{2}=x_{0}+\Delta_{2}$, so (6.6) is (to sufficient accuracy) the approximation

$$
z_{2}-p_{3}\left(z_{2}\right) / p_{3}^{\prime}\left(z_{2}\right)-\frac{1}{2} p_{3}^{2}\left(z_{2}\right) p_{3}^{\prime \prime}\left(z_{2}\right) /\left(p_{3}^{\prime}\left(z_{2}\right)\right)^{3}
$$

\section{Eighth-order methods}

If $k=m=1, n=3$, the relevant Jacobi polynomial is $G_{3}(2,2, x)=$ $\left(35 x^{3}-60 x^{2}+30 x-4\right) / 35$, with zeros

$$
\begin{aligned}
& \alpha=0.21234053823915294397 \ldots, \\
& \beta=0.59053313555926528913 \ldots,
\end{aligned}
$$

and

$$
\gamma=0.91141204048729605260 \ldots
$$

Method 113a.

$$
\alpha_{1}=\alpha
$$

where $\alpha$ is given by (6.7),

$$
\begin{gathered}
\Delta_{1}, f_{1}^{(1)}, \Delta_{2}, \text { and } \alpha_{2,1} \text { as for method } 112 \mathrm{a}, \\
a=100 \alpha_{2,1}^{2}-120 \alpha_{2,1}+30, \\
b=60 \alpha_{2,1}^{2}-75 \alpha_{2,1}+20, \\
c=30 \alpha_{2,1}^{2}-40 \alpha_{2,1}+12, \\
\alpha_{2,2}=\left(b-\left(b^{2}-a c\right)^{\frac{1}{2}}\right) / a, \\
f_{2}^{(1)}, t_{1}, t_{2}, v_{1}, v_{2}, \Delta_{3}, \text { and } \Delta_{4} \text { as for method } 112 \mathrm{a}, \\
\Delta_{5}=\Delta_{2}-\Delta_{3} / \Delta_{4}, \\
\alpha_{3.1}=\alpha_{1} \Delta_{1} / \Delta_{5}, \\
\alpha_{3,2}=\alpha_{2,2} \Delta_{2} / \Delta_{5},
\end{gathered}
$$




$$
\begin{aligned}
& \alpha_{3,3}=\frac{12-15\left(\alpha_{3,1}+\alpha_{3,2}\right)+20 \alpha_{3,1} \alpha_{3.2}}{15-20\left(\alpha_{3.1}+\alpha_{3,2}\right)+30 \alpha_{3,1} \alpha_{3,2}} \\
& f_{3}^{(1)}=f^{\prime}\left(x_{0}+\alpha_{3.3} \Delta_{5}\right), \\
& t_{3}=\left(f_{3}^{(1)}-f_{0}^{(1)}\right) /\left(\alpha_{3.3} \Delta_{s}\right) \\
& a_{1,1}=g\left(\alpha_{3,1}, \alpha_{3,2}, \alpha_{3,3}\right) \equiv \frac{6 \alpha_{3,2} \alpha_{3,3}-4\left(\alpha_{3,2}+\alpha_{3,3}\right)+3}{12\left(\alpha_{3,2}-\alpha_{3,1}\right)\left(\alpha_{3,3}-\alpha_{3,1}\right)}, \\
& a_{1,2}=g\left(\alpha_{3,2}, \alpha_{3,3}, \alpha_{3,1}\right) \text {, } \\
& a_{1,3}=g\left(\alpha_{3,3}, \alpha_{3,1}, \alpha_{3,2}\right) \text {, } \\
& a_{2,1}=h\left(\alpha_{3,1}, \alpha_{3,2}, \alpha_{3,3}\right) \equiv \frac{\left(1-\alpha_{3,2}\right)\left(1-\alpha_{3,3}\right)}{\left(\alpha_{3,2}-\alpha_{3,1}\right)\left(\alpha_{3,3}-\alpha_{3,1}\right)}, \\
& a_{2,2}=h\left(\alpha_{3,2}, \alpha_{3,3}, \alpha_{3.1}\right) \text {, } \\
& a_{2,3}=h\left(\alpha_{3,3}, \alpha_{3,1}, \alpha_{3,2}\right), \\
& {\left[\begin{array}{l}
\Delta_{6} \\
\Delta_{7}
\end{array}\right]=\left[\begin{array}{lll}
a_{1,1} & a_{1,2} & a_{1,3} \\
& & \\
a_{2,1} & a_{2,2} & a_{2,3}
\end{array}\right]\left[\begin{array}{l}
t_{1} \\
t_{2} \\
t_{3}
\end{array}\right],} \\
& \Delta_{8}=f_{0}^{(0)}+f_{0}^{(1)} \Delta_{5}+\Delta_{5}^{2} \Delta_{6} \\
& \Delta_{9}=f_{0}^{(1)}+\Delta_{s} \Delta_{7},
\end{aligned}
$$

and

$$
x_{1}=x_{0}+\Delta_{5}-\Delta_{8} / \Delta_{9}-\frac{1}{2} \Delta_{8}^{2} v_{1} / \Delta_{9} .
$$

The error constant is

$$
\begin{aligned}
K(\alpha, \beta, \gamma)= & {\left[3 \phi_{8}-21 \phi_{2} \phi_{7} /(1-\alpha)\right.} \\
& +9(35(1-\gamma)-3 /(1-\beta)) \phi_{3} \phi_{6} \\
& \left.-25\left(9-44 \gamma+42 \gamma^{2}\right) \phi_{4} \phi_{5}\right] / 3675
\end{aligned}
$$

\section{Comments on method 113a}

It is easy to verify that the polynomial

$$
\left(100 t^{2}-120 t+30\right) x^{2}-2\left(60 t^{2}-75 t+20\right) x+\left(30 t^{2}-40 t+12\right)
$$

is orthogonal to 1 and $x$ with respect to the weight function $x(x-t)$ on $[0,1]$. In view of (2.17), this explains (6.9). It may also be verified that, if $\alpha_{2.2}$ is defined by (6.9), then $b^{2}>a c$ for all real $\alpha_{2,1}$, and $\alpha_{2,2} \rightarrow \beta$ as $\alpha_{2,1} \rightarrow \alpha$. 


$$
\begin{aligned}
& \text { Similarly, } \\
& \int_{0}^{1}[(15-20(t+u)+30 t u) x-(12-15(t+u)+20 t u)] x(x-t)(x-u) d x=0,
\end{aligned}
$$

explaining (6.10), and $\alpha_{3,3} \rightarrow \gamma$ as $\alpha_{3,1} \rightarrow \alpha$ and $\alpha_{3,2} \rightarrow \beta$.

Methods 113a-113f. By taking $\alpha_{1}=\alpha, \beta$ or $\gamma$ in (6.8), and either sign before the square root in (6.9), we get six different methods, one of which is method 113a. Table 6.2 summarizes these methods. The error constants

$$
K=A \phi_{8}+B \phi_{2} \phi_{7}+C \phi_{3} \phi_{6}+D \phi_{4} \phi_{5}
$$

are obtained by suitably permuting $\alpha, \beta$ and $\gamma$ in (6.11). Numerical values of $A$, $B, C$ and $D$ are given in the table.

\begin{tabular}{|c|c|c|c|c|c|c|c|}
\hline Method & $\alpha_{1}$ & $\begin{array}{c}\text { Sign in } \\
(6.9)\end{array}$ & $\begin{array}{c}\text { Error } \\
\text { Constant }\end{array}$ & $A$ & $B$ & $C$ & $D$ \\
\hline $113 a$ & $\alpha$ & - & $K(\alpha, \beta, \gamma)$ & 0.000816 & -0.007255 & -0.010349 & -0.025756 \\
\hline $113 \mathrm{~b}$ & $\alpha$ & + & $K(\alpha, \gamma, \beta)$ & 0.000816 & -0.007255 & -0.047837 & 0.015897 \\
\hline $113 c$ & $\beta$ & - & $K(\beta, \gamma, \alpha)$ & 0.000816 & -0.013955 & -0.015420 & -0.010549 \\
\hline $113 d$ & $\beta$ & + & $K(\beta, \alpha, \gamma)$ & 0.000816 & -0.013955 & -0.001734 & -0.025756 \\
\hline $113 \mathrm{e}$ & $\gamma$ & - & $K(\gamma, \alpha, \beta)$ & 0.000816 & -0.064504 & 0.025770 & 0.015897 \\
\hline $113 \mathrm{f}$ & $\gamma$ & + & $K(\gamma, \beta, \alpha)$ & 0.000816 & -0.064504 & 0.049571 & -0.010549 \\
\hline
\end{tabular}

TABLE 6.2

Some eighth-order methods

\section{Comparison of error constants}

It is natural to ask which of methods $113 \mathrm{a}-113 \mathrm{f}$ has minimal error constant $|K|$. From (6.11), this depends on the behaviour of $\phi_{2}, \cdots, \phi_{8}$. Suppose that $f$ is holomorphic in a closed disk $|z-\zeta| \leqq r$, so there is a constant $c$ such that

$$
\left|\phi_{j}\right| \leqq c r^{1-j}
$$

for all $j \geqq 2$. (Conversely, if (6.13) holds then $f$ is holomorphic in the open disk $|z-\zeta|<t$.) From (6.12) and (6.13),

$$
|K| \leqq(|A|+|B|+|C|+|D|) c r^{-7},
$$

so a reasonable criterion is to choose the method with minimal $\sigma=$ $|A|+|B|+|C|+|D|$. (A similar but slightly different criterion is given by King 
[16].) On our criterion, method $113 \mathrm{c}(\sigma=0.0399)$ is slightly better than methods $113 \mathrm{~d}(\sigma=0.0414)$ and $113 \mathrm{a}(\sigma=0.0434)$, but the difference is small. On the same criterion, method $112 \mathrm{a}$ is better than method $112 \mathrm{~b}$.

\section{A seventh-order method}

If $k=1, m=n=2$, the relevant Jacobi polynomial is $G_{2}(3,3, x)=$ $x^{2}-4 x / 3+2 / 5$, with zeros $(10 \pm \sqrt{10}) / 15$. By Theorem 2.1 , methods in $B(1,2,2)$ have order 7 . One such method is the following.

METHOD 122.

$$
\begin{aligned}
& \alpha_{1}=(10-\sqrt{10}) / 15, \quad \alpha_{2}=(10+\sqrt{10}) / 15, \\
& \Delta_{1}=-f_{0}^{(0)} / f_{0}^{(1)}, \quad \Delta_{2}=\Delta_{1}-\frac{1}{2} f_{0}^{(2)} \Delta_{1}^{2} / f_{0}^{(1)}, \\
& f_{1}^{(1)}=f^{\prime}\left(x_{0}+\alpha_{1} \Delta_{2}\right), \quad f_{2}^{(1)}=f^{\prime}\left(x_{0}+\alpha_{2} \Delta_{2}\right),
\end{aligned}
$$

$x_{1}$ an approximate zero of $p_{3}$, satisfying (2.3) and

$$
p_{3}\left(x_{1}\right)=O\left(\delta^{8}\right) \text {. }
$$

(An explicit formula for $x_{1}$, similar to those above, is easy to derive.)

Provided (6.15) holds, instead of merely (2.4), the error constant is $-\phi_{7} / 225-2 \phi_{2}^{2} \phi_{5} / 3+\phi_{3} \phi_{5} / 3$. Unlike some of the methods above, method 122 remains the same when $\alpha_{1}$ and $\alpha_{2}$ are interchanged.

\section{Other methods}

The obvious method which uses evaluations of $f, f^{\prime}$ and $f^{\prime \prime}$ at $x_{0}$, followed by evaluations of $f^{\prime}$ and $f^{\prime \prime}$ at another point $y_{1}=x_{0}+O(\delta)$, has order five. It is natural to ask if there is a choice of $y_{1}$ for which the order is six. Theorems 2.1 and 4.1 are not applicable, but for a similar analysis to go through we need a nonzero number $\alpha$ such that $P(1)=0$, for all fifth-degree polynomials $P(x)$ satisfying $P(0)=P^{\prime}(0)=P^{\prime \prime}(0)=P^{\prime}(\alpha)=P^{\prime \prime}(\alpha)=0$ (compare Lemma 4.3). This condition gives

$$
\operatorname{det}\left[\begin{array}{ccc}
1 & 1 & 1 \\
3 & 4 \alpha & 5 \alpha^{2} \\
6 & 12 \alpha & 20 \alpha^{2}
\end{array}\right]=0
$$

which (using $\alpha \neq 0$ ) reduces to

$$
10 \alpha^{2}-15 \alpha+6=0 .
$$

Since (7.2) has no real roots, there is no real sixth-order method of this type. 
Similarly, it is natural to ask if there is an eighth-order method which uses evaluations of $f, f^{\prime}, f^{\prime \prime}$, and $f^{\prime \prime \prime}$ at $x_{0}$, followed by evaluations of $f^{\prime}, f^{\prime \prime}$ and $f^{\prime \prime \prime}$ at some point $y_{1}$. In this case we need a nonzero $\alpha$ satisfying

which reduces to

$\operatorname{det}\left[\begin{array}{cccc}1 & 1 & 1 & 1 \\ 4 & 5 \alpha & 6 \alpha^{2} & 7 \alpha^{3} \\ 12 & 20 \alpha & 30 \alpha^{2} & 42 \alpha^{3} \\ 24 & 60 \alpha & 120 \alpha^{2} & 210 \alpha^{3}\end{array}\right]=0$

$$
35 \alpha^{3}-84 \alpha^{2}+70 \alpha-20=0
$$

and (7.4) has one real root $\alpha=0.74494327207110343664 \ldots$ We shall not give the details of this method, but note that, provided the polynomial approximations are solved sufficiently accurately, the error constant is simply $(1-\alpha)^{4} \phi_{8}$. Some numerical results for this method $\left(S_{8}\right)$ are given in Section 9.

These examples suggest several questions. For example, there are methods of order $2 m+1$ which use evaluations of $f\left(x_{0}\right), \cdots, f^{(m)}\left(x_{0}\right)$ and $f^{\prime}\left(y_{1}\right)$, $\cdots, f^{(m)}\left(y_{1}\right)$, but for which $m$ are there (real) methods of order $2 m+2$ ? (There are such methods for $m=1,3,5$, etc., but none is known for even $m$.) Similarly, for which $n$ are there methods of order $3(n+1)$ using evaluations of $f\left(x_{0}\right)$, $f^{\prime}\left(x_{0}\right), f^{\prime \prime}\left(x_{0}\right)$ and $f^{\prime}\left(y_{i}\right), f^{\prime \prime}\left(y_{i}\right)$ for suitable real points $y_{1}, \cdots, y_{n}$ ? Some recent results are given in [38] and [39].

A possible extension of our results is to methods where the evaluation of derivatives $f^{(k)}\left(y_{i}\right)$ is replaced by the evaluation of definite integrals

$$
f^{(-1)}\left(y_{i}\right)=\int_{x_{0}}^{y_{1}} f(t) d t, f^{(-2)}\left(y_{i}\right)=\int_{x_{0}}^{y_{i}}\left(\int_{x_{0}}^{u} f(t) d t\right) d u=\int_{x_{0}}^{y_{i}}\left(y_{i}-t\right) f(t) d t,
$$

etc. For example, if $m \geqq 1, n=1$ and $0<k \leqq m+1$, our theory gives

$$
\alpha_{1}=\frac{m+2-k}{m+2} \text {. }
$$

It is suggestive that the fourth-order of [40] have $\alpha_{1}=1$, which is obtained formally by setting $k=0$ in (7.5). Similarly, $\alpha_{1}=(m+3) /(m+2)$ is obtained formally by setting $k=-1$ in (7.5), and there is in fact a method of order $m+3$ which uses evaluations of $f\left(x_{0}\right), \cdots, f^{(m)}\left(x_{0}\right)$ and $f^{(-1)}\left(y_{1}\right)$, where $y_{1}$ is determined in the usual way from this value of $\alpha_{1}$. (For details of this method, see Kacewicz [15] and Wozniakowski [35].) However, we do not expect the formal analogy to hold for large $n$. One reason for this is that an order of at least $2^{\ln / 21}$ is attainable by methods using one evaluation of $f$ and $n$ evaluations of 
$f^{(-1)}$ per iteration, for two evaluations of $f^{(-1)}$ can be used to approximate one evaluation of $f$ to any desired accuracy. Note that finding a zero of $f$ using evaluations of $f^{(-1)}$ is equivalent to finding a turning point of $g=f^{(-1)}$ using evaluations of $g$, so the methods discussed in Brent [3] may also be used.

\section{A class of nonlinear Runge-Kutta methods}

Consider the ordinary differential equation

$$
d x / d t=g(x),
$$

with initial condition $x\left(t_{0}\right)=x_{0}$. If $t_{1}=t_{0}+h$, and we want to find $x\left(t_{1}\right)$, we need a zero of the function

$$
f(x)=\int_{x_{0}}^{x} \frac{d u}{g(u)}-h
$$

Since $f^{\prime}(x)=1 / g(x)$ may be computed almost as easily as $g(x)$, and $f\left(x_{0}\right)=-h$ is known without any computation, a zero-finding method using evaluations of $f\left(x_{0}\right), f^{\prime}\left(x_{0}\right), f^{\prime}\left(y_{1}\right), \cdots, f^{\prime}\left(y_{n}\right)$ is applicable. For example, one iteration of method 111 a (see Section 6) may be written as

and

$$
\begin{aligned}
& g_{0}=g\left(x_{0}\right), \\
& \Delta_{1}=h g_{0}, \\
& g_{\alpha}=g\left(x_{0}+2 \Delta_{1} / 3\right),
\end{aligned}
$$

$$
x_{1}=x_{0}+\Delta_{1}\left(5+3\left(g_{\alpha} / g_{0}\right)^{2}\right) / 8
$$

when $f$ is defined by (8.2). The equations (8.3) give an explicit method of Runge-Kutta type for solving the differential equation (8.1). The method is "nonlinear" because the formula for $x_{1}$ is nonlinear in $g_{0}$ and $g_{\alpha}$. Since the zero-finding method is fourth-order, $x_{1}=x\left(t_{1}\right)+O\left(h^{4}\right)$, so the Runge-Kutta method (8.3) has order three. Note the difference in the definitions of order for differential equation methods (Henrici [11]) and methods for finding zeros.

Similarly, for any zero-finding method in $C(1,1, n)$, there is a corresponding nonlinear Runge-Kutta method. Numerical results for some of these methods are given in Section 9. By Theorem 4.1, the order of the zero-finding method is $2(n+1)$, so the order of of the Runge-Kutta method is $2 n+1$. Thus (with $\nu=n+1$ ) we have the following theorem, which is related to some results of $\mathrm{N} \not r$ rsett [25] and Osborne [27]. 
TheOREM 8.1. If $\nu>0$, there is an explicit, nonlinear, Runge-Kutta method of order $2 \nu-1$, using $\nu$ function evaluations per iteration, for single differential equations of the form (8.1).

Theorem 8.1 contrasts with the known results for (linear) Runge-Kutta methods for systems of differential equations of the form

$$
d x / d t=g(x, t) .
$$

From [9], the highest order attainable by such methods using $\nu$ evaluations of $g(x, t)$ per iteration is

$$
p^{\star}(\nu)=\left\{\begin{array}{ccc}
\nu & \text { if } & 1 \leqq \nu \leqq 4 \\
\nu-1 & \text { if } & 5 \leqq \nu \leqq 7 \\
\nu-2 & \text { if } & 8 \leqq \nu \leqq 9
\end{array}\right.
$$

and

$$
p^{\star}(\nu) \leqq \nu-2 \text { if } \nu \geqq 10 .
$$

Thus, it seems unlikely that a generalization of our nonlinear methods to systems of differential equations is possible, although an extension to single equations of the form $d x / d t=g(x, t)$ may be possible.

Examples of the use of our methods for the computation of inverse distribution functions are given below, and in [7].

\section{Numerical results}

In this section we summarize the results of numerical tests of some of the methods described in Sections 6 to 8. Table 9.1 gives $\varepsilon_{i}=x_{i}-\zeta(i=1, \cdots, 4)$ for the function

$$
f(x)=x^{2}-x-3+4 / x-\log _{2} x,
$$

with a simple zero at $\zeta=2$, from the initial approximation $x_{0}=10$. Multipleprecision arithmetic was used to obtain $\varepsilon_{3}$ and $\varepsilon_{4}$ accurately in order to demonstrate the superlinear convergence. The order $\rho$ and asymptotic error constant $K$ are as given above.

All the methods converge, although $x_{0}$ is not very close to $\zeta$. The higher order methods give good approximations after two iterations (e.g. $\varepsilon_{2}=1.03^{\prime}-10$ for method 113a). Method 111d is the best of the fourth-order methods, at least for the function (9.1). 
TABLE 9.1

Numerical results for the function (9.1)

Method Order

$K$

$\varepsilon_{1}$

$\varepsilon_{2}$

$\varepsilon_{3}$

$\varepsilon_{4}$

\begin{tabular}{lllllll}
\hline $111 \mathrm{a}$ & 4 & 3.61 & 1.56 & $1.80^{\prime}-1$ & $1.33^{\prime}-3$ & $1.12^{\prime}-11$ \\
$111 \mathrm{~b}$ & 4 & 2.60 & 1.44 & $1.43^{\prime}-1$ & $5.02^{\prime}-4$ & $1.65^{\prime}-13$ \\
$111 \mathrm{c}$ & 4 & $3.32^{\prime}-1$ & $9.87^{\prime}-1$ & $4.09^{\prime}-2$ & $8.18^{\prime}-7$ & $1.49^{\prime}-25$ \\
$111 \mathrm{~d}$ & 4 & $3.32^{\prime}-1$ & $4.50^{\prime}-1$ & $3.53^{\prime}-3$ & $5.05^{\prime}-11$ & $2.16^{\prime}-42$ \\
$112 \mathrm{a}$ & 6 & $1.12^{\prime}-2$ & $3.86^{\prime}-1$ & $5.86^{\prime}-5$ & $4.55^{\prime}-28$ & $9.94^{\prime}-167$ \\
$113 \mathrm{a}$ & 8 & $3.69^{\prime}-4$ & $1.49^{\prime}-1$ & $1.03^{\prime}-10$ & $4.77^{\prime}-84$ & $9.81^{\prime}-671$ \\
122 & 7 & $6.90^{\prime}-2$ & $6.27^{\prime}-1$ & $1.79^{\prime}-3$ & $4.02^{\prime}-21$ & $1.17^{\prime}-144$ \\
$S_{8}$ & 8 & $2.82^{\prime}-5$ & $6.44^{\prime}-1$ & $2.66^{\prime}-3$ & $4.52^{\prime}-21$ & $4.94^{\prime}-168$ \\
\hline
\end{tabular}

Table 9.2 illustrates equation (6.1). For $f$ given by (9.1) and various $\varepsilon_{0}=x_{0}-\zeta$, the table gives the computed values $K\left(\varepsilon_{0}\right)=\varepsilon_{1} / \varepsilon_{0}^{p}$, and the predicted asymptotic error constant $K=\lim _{\varepsilon_{0} \rightarrow 0} K\left(\varepsilon_{0}\right)$. The agreement between the predicted and computed values is good.

TABLE 9.2

Computed and predicted error constants

\begin{tabular}{lccccc}
\hline Method Order & $K\left(10^{-4}\right)$ & $K\left(10^{-8}\right)$ & $K\left(10^{-12}\right)$ & $K$ \\
\hline & & & & & \\
$112 \mathrm{a}$ & 6 & $1.12131^{\prime}-2$ & $1.12045^{\prime}-2$ & $1.12045^{\prime}-2$ & $1.12045^{\prime}-2$ \\
$113 \mathrm{a}$ & 8 & $3.68987^{\prime}-4$ & $3.68889^{\prime}-4$ & $3.68889^{\prime}-4$ & $3.6889^{\prime}-4$ \\
122 & 7 & $6.89218^{\prime}-2$ & $6.89766^{\prime}-2$ & $6.89766^{\prime}-2$ & $6.89766^{\prime}-2$ \\
$S_{8}$ & 8 & $7.11402^{\prime}-2$ & $3.53173^{\prime}-5$ & $2.81896^{\prime}-5$ & $2.81889^{\prime}-5$ \\
\hline
\end{tabular}

Finally, Table 9.3 gives numerical results for some of the Runge-Kutta methods described in Section 8 and some more usual Runge-Kutta methods. Suppose we want to tabulate solutions of

$$
(2 \pi)^{-\frac{1}{2}} \int_{0}^{x} e^{-u^{2} / 2} d u=t
$$


for $t=0.0(0.1) 0.4$ or $t=0.0(0.01) 0.49$. Equivalently, we want to solve the differential equation

$$
d x / d t=(2 \pi)^{\frac{1}{2}} e^{x^{2 / 2}}
$$

with initial condition $x(0)=0$. The following methods are possible:

1. Numerical integration of the left side of (9.2), followed by interpolation. This would be appropriate if the solution were to be tabulated for given values of $x$, but it is inconvenient if the solution is required for given values of $t$.

2. Using some method for second-order differential equations (or systems of first order equations) applied to the equation $d^{2} x / d t^{2}=x(d x / d t)^{2}$ with appropriate initial conditions. This avoids the repeated evaluation of exponentials, but depends on special properties of the integrand in (9.2), so is not generally applicable.

3. Using some method for first-order differential equations applied to (9.3). We compare some such methods.

In Table 9.3, method $111 \mathrm{~d}^{\prime}$ is the (third-order) nonlinear Runge-Kutta method derived from the (fourth-order) zero-finding method $111 \mathrm{~d}$ as described in Section 8 , and similarly for $112 \mathrm{a}^{\prime}$ and $113 \mathrm{a}^{\prime}$. Method $R K 4$ is the classical fourth-order method of Kutta [23], and $R K 7$ is the seventh-order method of Shanks [30]. (The use of method $R K 4$ to solve nonlinear equations was suggested by Kizner [19].) The number of evaluations of $e^{x^{2 / 2}}$ per iteration is denoted by $\nu$. If $\hat{x}_{h}(t)$ is the computed solution (using step size $h$ ), the error $e_{h}(t)$ is defined by

$$
e_{h}(t)=(2 \pi)^{-\frac{1}{2}} \int_{0}^{\bar{x}_{h}(t)} e^{-u^{2} / 2} d u-t
$$

Computations were performed with double-precision floating-point arithmetic on a Univac 1108 computer (fraction length 60 bits).

The table suggests that our methods are more accurate than standard Runge-Kutta methods with the same number of function evaluations per iteration, and more efficient than standard methods with the same order. For example, method 113a' is considerably more accurate than $R K 4$, though both methods require four function evaluations per iteration; and 113a' is slightly more accurate than $R K 7$, which requires nine function evaluations per iteration. This is not surprising, for our methods are applicable only to single differential equations of the special form (8.1), but the standard methods are applicable to general systems of the form (8.4). 
TABLE 9.3

Comparison of Runge-Kutta methods

\begin{tabular}{lcccccc}
\hline Method & $\nu$ & Order & $e_{0},(0.2)$ & $e_{001}(0.2)$ & $e_{0},(0.4)$ & $e_{001}(0.4)$ \\
\hline $111 \mathrm{~d}^{\prime}$ & 2 & 3 & $-4.59^{\prime}-5$ & $-5.66^{\prime}-8$ & $-9.45^{\prime}-6$ & $1.49^{\prime}-7$ \\
$112 \mathrm{a}^{\prime}$ & 3 & 5 & $1.22^{\prime}-7$ & $2.54^{\prime}-12$ & $3.16^{\prime}-6$ & $-2.47^{\prime}-11$ \\
$113 \mathrm{a}^{\prime}$ & 4 & 7 & $-6.28^{\prime}-10$ & $-6.29^{\prime}-17$ & $3.86^{\prime}-8$ & $3.69^{\prime}-15$ \\
$R K 4$ & 4 & 4 & $-3.74^{\prime}-7$ & $2.12^{\prime}-11$ & $1.95^{\prime}-5$ & $7.90^{\prime}-9$ \\
$R K 7$ & 9 & 7 & $-1.76^{\prime}-9$ & $-2.86^{\prime}-16$ & $-5.19^{\prime}-7$ & $-1.67^{\prime}-13$ \\
\hline
\end{tabular}

\section{Acknowledgement}

I wish to thank J. C. Butcher, M. R. Osborne and J. F. Traub for several useful comments on earlier versions of this paper.

\section{References}

[1] M. Abramowitz and I. A. Stegun, Handbook of Mathematical Functions with Formulas, Graphs, and Mathematical Tables, National Bureau of Standards, Washington, D. C., (1964).

[2] C. W. R. de Boor and B. Swartz, 'Collocation at Gaussian points', SIAM J. Numer. Anal., 10 (1973), 582-606.

[3] R. P. Brent, Algorithms for Minimization without Derivatives, Prentice-Hall, Englewood Cliffs, New Jersey (1973).

[4] R. P. Brent, 'Some efficient algorithms for solving systems of nonlinear equations', SIAM J. Numer. Anal., 10 (1973), 327-344.

[5] R. P. Brent, Efficient methods for finding zeros of functions whose derivatives are easy to evaluate, Computer Science Dept., Carnegie-Mellon Univ., Pittsburgh, Pennsylvania, (1974).

[6] R. P. Brent, Computer Solution of Nonlinear Equations, Academic Press, (to appear).

[7] R. P. Brent and E. M. Brent, 'Efficient computation of inverse distribution functions', (in preparation).

[8] R. P. Brent, S. Winograd and P. Wolfe, 'Optimal iterative processes for root-finding', Numer. Math. 20 (1973), 327-341.

[9] J. C. Butcher, 'On the attainable order of Runge-Kutta methods', Math. Comp., 19 (1965), $408-417$.

[10] P. J. Davis, Interpolation and Approximation, Blaisdell, New York, (1963).

[11] P. Henrici, Discrete Variable Methods in Ordinary Differential Equations, Wiley, New York, (1962).

[12] E. Isaacson and H. B. Keller, Analysis of Numerical Methods, Wiley, New York, (1966).

[13] P. Jarratt, 'Some efficient fourth order multipoint methods for solving equations', BIT 9 (1969), 11-124.

[14] P. Jarratt, 'A review of methods for solving nonlinear algebraic equations in one variable' in Numerical Methods for Nonlinear Algebraic Equations (P. Rabinowitz, ed.), Gordon and Breach, New York, (1970), pp.1-26.

[15] B. Kacewicz, 'An integral-interpolatory iterative method for the solution of scalar equations', Computer Sci. Dept., Carnegie-Mellon Univ., Pittsburg, Pennsylvania, (1975).

[16] R. F. King, 'Runge-Kutta methods with constrained minimum error bounds', Math. Comp., 20 (1966), 386-391. 
[17] R. F. King, 'A fifth-order family of modified Newton methods', BIT 11 (1971), 409-412.

[18] R. F. King, 'Tangent methods for nonlinear equations', Numer. Math. 18 (1972), 298-304.

[19] W. Kizner, 'A numerical method for finding solutions of nonlinear equations', J. SIAM 12 (1964), 424-428.

[20] H. T. Kung and J. F. Traub, 'Optimal order of one-point and multipoint iteration', J. ACM, 21 (1974), 643-651.

[21] H. T. Kung and J. F. Traub, 'Computational complexity of one-point and multipoint iteration', in Complexity of Real Computation (R. Karp, ed.) American Math. Soc., New York, (1973).

[22] H. T. Kung and J. F. Traub, 'Optimal order and efficiency for iterations with two evaluations', Computer Sci. Dept., Carnegie-Mellon Univ., Pittsburgh, Pennsylvania, (1973).

[23] W. Kutta, 'Beitrag zur näherungsweisen Integration totaler Differentialgleichungen', Z. Math. Phys. 46 (1901), 435-452.

[24] C. A. Micchelli and T. J. Rivlin, 'Numerical integration rules near Gaussian quadrature', Report RC4387, IBM Research Center, Yorktown Heights, New York, (1973).

[25] S. P. Nørsett, 'One-step methods of Hermite type for numerical integration of stiff systems', BIT 14 (1974), 63-77.

[26] J. M. Ortega and W. C. Rheinboldt, Iterative Solution of Nonlinear Equations in Several Variables, Academic Press, New York, (1970).

[27] M. R. Osborne, 'Minimising truncation error in finite difference approximations to ordinary differential equations', Math. Comp. 21 (1967), 135-145.

[28] M. R. Osborne, 'Collocation, difference equations, and stitched function representations', in Topics in Numerical Analysis (J. J. H. Miller, ed., Academic Press, (1974).

[29] A. M. Ostrowski, Solution of Equations in Euclidean and Banach Spaces (3rd ed. of Solution of Equations and Systems of Equations), Academic Press, New York, (1973).

[30] E. B. Shanks, 'Solutions of differential equations by evaluations of functions', Math. Comp. 20 (1966), 21-38.

[31] G. Szegö, Orthogonal Polynomials, AMS Colloquium Publication, Vol. 23 (1959), American Math. Soc., New York.

[32] J. F. Traub, Iterative Methods for the Solution of Equations, Prentice-Hall, Englewood Cliffs, New Jersey, (1964).

[33] J. H. Wilkinson, Rounding Errors in Algebraic Processes, HMSO, London, (1963).

[34] H. Wozniakowski, 'Maximal stationary iterative methods for the solution of operator equations', SIAM J. Numer. Anal., 11 (1974a), 934-949.

[35] H. Wozniakowski, 'Generalized information and maximal order of iteration for operator equations', SIAM J. Numer. Anal., 12 (1975), 121-135.

[36] H. Wozniakowski, Properties of maximal order methods for the solution of equations, Computer Science Dept., Carnegie-Mellon Univ., Pittsburgh, Pennsylvania, (1975).

[37] H. Wozniakowski, Private Communication, (1975b) (January).

[38] R. P. Brent, 'A class of optimal-order zero-finding methods using derivative evaluations', in Analytic Computational Complexity (J. F. Traub, ed.), Academic Press, New York, (1975).

[39] R. Meersman, 'Optimal use of information in certain iterative processes', in Analytic Computational Complexity (J. F. Traub, ed.), Academic Press, New York, (1975).

\section{Computer Centre,}

Australian National University,

Canberra, Australia. 